\title{
Holographic Phase Transition Probed by Nonlocal Observables
}

\author{
Xiao-Xiong Zeng ${ }^{1,2}$ and Li-Fang $\mathrm{Li}^{3}$ \\ ${ }^{1}$ School of Material Science and Engineering, Chongqing Jiaotong University, Chongqing 400074, China \\ ${ }^{2}$ State Key Laboratory of Theoretical Physics, Institute of Theoretical Physics, Chinese Academy of Sciences, Beijing 100190, China \\ ${ }^{3}$ Center for Space Science and Applied Research, Chinese Academy of Sciences, Beijing 100190, China
}

Correspondence should be addressed to Xiao-Xiong Zeng; xxzeng@mail.bnu.edu.cn

Received 31 March 2016; Accepted 8 June 2016

Academic Editor: Davood Momeni

Copyright (C) 2016 X.-X. Zeng and L.-F. Li. This is an open access article distributed under the Creative Commons Attribution License, which permits unrestricted use, distribution, and reproduction in any medium, provided the original work is properly cited. The publication of this article was funded by $\mathrm{SCOAP}^{3}$.

From the viewpoint of holography, the phase structure of a 5-dimensional Reissner-Nordström-AdS black hole is probed by the two-point correlation function, Wilson loop, and entanglement entropy. As the case of thermal entropy, we find for all the probes that the black hole undergoes a Hawking-Page phase transition, a first-order phase transition, and a second-order phase transition successively before it reaches a stable phase. In addition, for these probes, we find that the equal area law for the first-order phase transition is valid always and the critical exponent of the heat capacity for the second-order phase transition coincides with that of the mean field theory regardless of the size of the boundary region.

\section{Introduction}

Phase transition is a ubiquitous phenomenon for gardenvariety thermodynamic systems. Due to the pioneering work by Hawking $[1,2]$, a black hole is also a thermodynamic system. Such a fact is further supported by AdS/CFT correspondence [3-5], where a black hole in the AdS bulk is dual to a thermal system without gravity. So one can naturally expect that a black hole can also undertake some interesting phase transitions as the general thermodynamic system. Actually it has been shown that a charged AdS black hole undergoes a Hawking-Page phase transition [6,7], which is interpreted as the confinement/deconfinement phase transition in the dual gauge field theory [8] and a van der Waals-like phase transition before it reaches the stable state [9]. The Hawking-Page phase transition implies that the thermal AdS is unstable and it will transit to the stable Schwarzschild AdS black hole lastly. The van der Waals-like phase transition has been observed till now in many circumstances. The first observation was contributed by [9] in the $T-S$ plane. Specifically speaking, in a fixed charge ensemble, for a black hole endowed with small charge, there is an unstable black hole interpolating between the stable small hole and stable large hole, and the small stable hole will undertake a first-order phase transition to the large stable hole as the temperature of the black hole reaches a critical temperature. As the charge increases to the critical charge, the small hole and the large hole merge into one and squeeze out the unstable phase so that an inflection point emerges and the phase transition is second order. When the charge exceeds the critical charge, the black hole is always stable. Recently in the extended phase space, where the negative cosmological constant is treated as the pressure while its conjugate acts as the thermodynamical volume, the van der Waals-like phase transition has also been observed in the $P-V$ plane [10-16]. In addition, it was shown in [17] that the van der Waals-like phase transition also shows up in the $Q-\Phi$ plane. Particularly, in the Gauss-Bonnet gravity, it is found that the Gauss-Bonnet coupling parameter $\alpha$ also affects the phase structure of the space time, and in the $T-\alpha$ plane, a 5-dimensional neutral Gauss-Bonnet black hole also demonstrates the van der Waals-like phase transition [18].

In this paper, we intend to probe the Hawking-Page phase transition and van der Waals-like phase transition appeared in a 5-dimensional Reissner-Nordström-AdS black hole by the geodesic length, minimal area surface, and minimal surface area in the bulk, which are dual to the nonlocal observables on the boundary theory by holography, namely, the twopoint correlation function, Wilson loop, and entanglement 
entropy, individually (recently these nonlocal observables have been used to probe the nonequilibrium thermalization process, and it has been found that all of them have the same effect [19-25]). In fact, there have been some similar works to probe the phase structure by holographic entanglement entropy. In [26], the phase structure of entanglement entropy is studied in the $T-S$ plane for both a fixed charge ensemble and a fixed chemical potential ensemble, and it is found that the phase structure of entanglement entropy is similar to that of the thermal entropy. In particular, the entanglement entropy is found to demonstrate the same second-order phase transition at the critical point as the thermal entropy. Soon after, it is found that the entanglement entropy can also probe the van der Waals-like phase transition in the $P-V$ plane [27]. In [28], Nguyen has investigated exclusively the equal area law of holographic entanglement entropy and found that the equal area law holds regardless of the size of the entangling region. Very recently [29] investigated entanglement entropy for a quantum system with infinite volume; their result showed that the entanglement entropy also exhibits the same van der Waals-like phase transition as the thermal entropy. They also checked the equal area law and obtained the critical exponent of the heat capacity near the critical point.

In this paper, we will further investigate whether one can probe the phase structure by two-point correlation function and Wilson loop besides the entanglement entropy. We intend to explore whether they exhibit the similar van der Waals-like phase transition as the entanglement entropy and thermal entropy. In addition, we also want to check whether these nonlocal observables can probe the Hawking-Page phase transition between the AdS black hole and thermal gas so that we can get a complete picture about the phase transition of the black holes in the framework of holography.

This paper is organized as follows. In Section 2, we will discuss the thermal entropy phase transition of a 5dimensional Reissner-Nordström-AdS black hole in the $T-S$ plane in a fixed charge ensemble. Then in Section 3, we will probe these phase transitions by geodesic length, Wilson loop, and holographic entanglement entropy individually. In each subsection, the equal area law is checked and the critical exponent of the heat capacity is obtained for different sizes of the boundary region. Section 4 is devoted to discussions and conclusions.

\section{Thermodynamic Phase Transition of the 5-Dimensional Reissner-Nordström- AdS Black Hole}

Starting from the action

$$
S=\frac{1}{16 \pi G} \int d^{n+1} x \sqrt{-g}\left[R-\frac{1}{4} F_{\mu \nu} F^{\mu \nu}+\frac{n(n-1)}{l^{2}}\right],
$$

where $F_{\mu \nu}=\partial_{\mu} A_{\nu}-\partial_{\nu} A_{\mu}$ and $l$ is the AdS radius, we shall focus on the case of $n=4$, in which the charged ReissnerNordström-AdS black hole can be written as [9]

$$
\begin{aligned}
d s^{2}= & -f(r) d t^{2}+f^{-1}(r) d r^{2} \\
& +r^{2}\left[d \phi^{2}+\sin ^{2} \phi\left(d \theta^{2}+\sin ^{2} \theta d \psi^{2}\right)\right],
\end{aligned}
$$

where $\phi \in(0, \pi), \theta \in(0, \pi)$, and $\psi \in(0,2 \pi)$ are hyperspherical coordinates for the 3 spheres, and

$$
f(r)=1+\frac{r^{2}}{l^{2}}-\frac{8 M}{3 \pi r^{2}}+\frac{4 Q^{2}}{3 \pi^{2} r^{4}},
$$

with $M$ and $Q$ being the mass and charge of the black hole. Whence we can get the Hawking temperature of this space time as

$$
T=\left.\frac{f^{\prime}(r)}{4 \pi}\right|_{r_{+}}=\frac{3 \pi^{2} r_{+}^{6}-8 l^{2}\left(Q^{2}-M \pi r_{+}^{2}\right)}{6 l^{2} \pi^{3} r_{+}^{5}} .
$$

In addition, it follows from the Bekenstein-Hawking formula that the entropy of the black hole is given by

$$
S=\frac{\pi^{2} r_{+}^{3}}{2}
$$

where $r_{+}$is the outer event horizon of the black hole, namely, the largest root of the equation $f\left(r_{+}\right)=0$. With this, the mass of the back hole can thus be expressed as the function of the event horizon:

$$
M=\frac{4 l^{2} Q^{2}+3 l^{2} \pi^{2} r_{+}^{4}+3 \pi^{2} r_{+}^{6}}{8 l^{2} \pi r_{+}^{2}}
$$

Substituting (5) and (6) into (4), we can get the relation between the temperature $T$ and entropy $S$ of the 5dimensional Reissner-Nordström-AdS black hole:

$$
T=\frac{12 S^{2}+l^{2}\left(-2 \pi^{2} Q^{2}+32^{1 / 3} \pi^{4 / 3} S^{4 / 3}\right)}{62^{2 / 3} l^{2} \pi^{5 / 3} S^{5 / 3}} .
$$

In addition, with the relation $F=M-T S$, the Helmholtz free energy can be expressed as

$$
F=\frac{5 Q^{2}}{6 \pi r_{+}^{2}}-\frac{1}{8} \pi r_{+}^{2}\left(-1+r_{+}^{2}\right) .
$$

Note that this formula for our free energy has implicitly chosen the pure AdS as the reference space time because the free energy vanishes for pure AdS by this formula. Now let us review the relevant phase transitions in the fixed charge ensemble by (7) and (8) in the $T$ - $S$ plane.

To achieve this, we should first find the critical charge by the following equations:

$$
\left(\frac{\partial T}{\partial S}\right)_{Q}=\left(\frac{\partial^{2} T}{\partial S^{2}}\right)_{Q}=0
$$

Inserting (7) into (9), we can get the values for the critical charge and critical entropy:

$$
\begin{aligned}
& Q_{c}=\frac{l^{2} \pi}{6 \sqrt{5}}, \\
& S_{c}=\frac{l^{3} \pi^{2}}{6 \sqrt{3}} .
\end{aligned}
$$




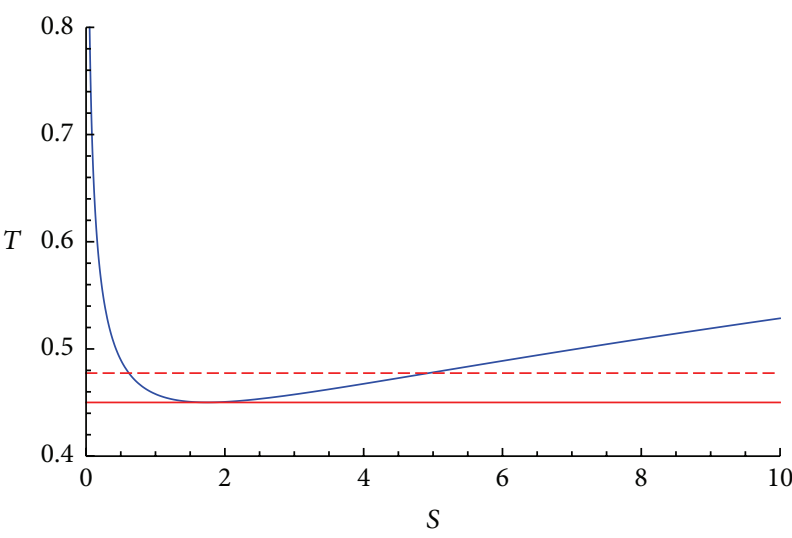

(a) $Q=0$

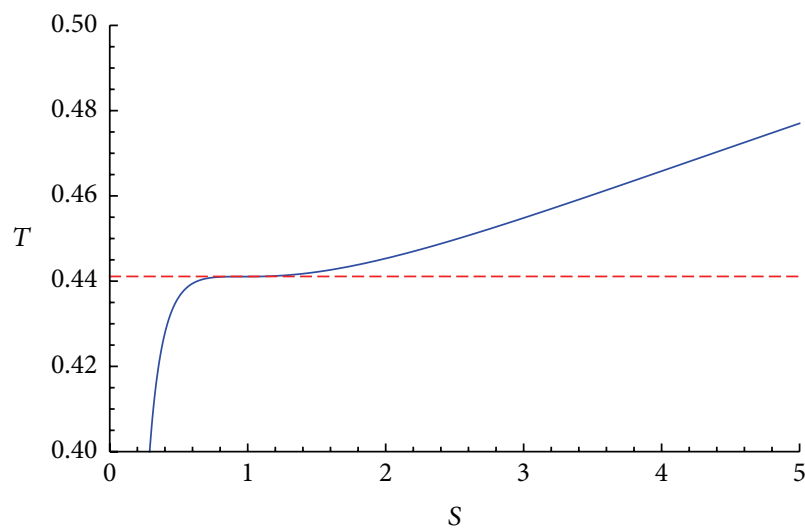

(c) $Q=\pi / 6 \sqrt{5}$

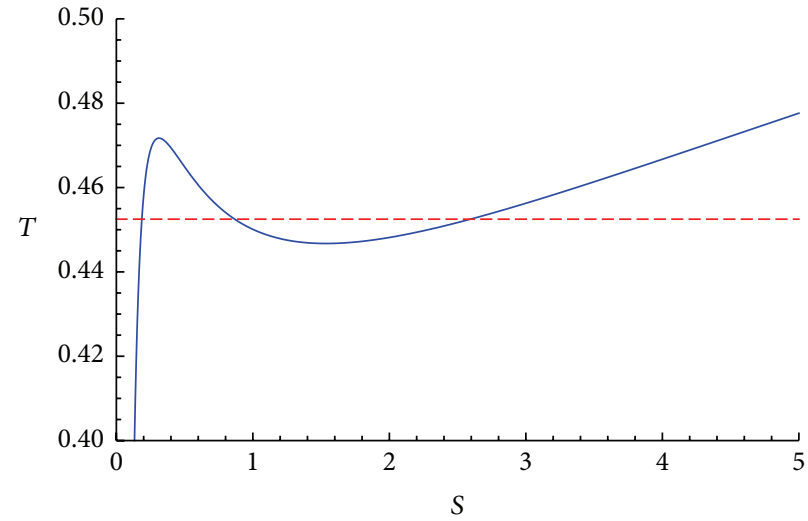

(b) $Q=(\pi-1) / 6 \sqrt{5}$

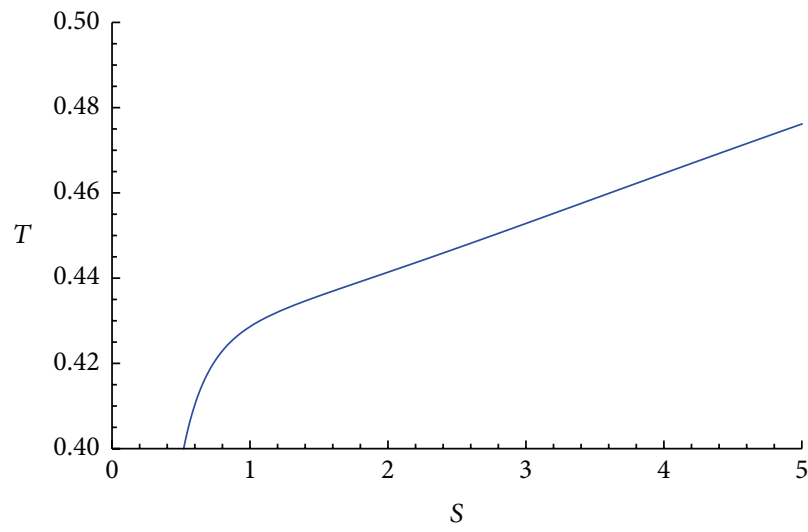

(d) $Q=(\pi+1) / 6 \sqrt{5}$

FIGURE 1: Relation between the entropy and temperature for different charges in the fixed charge ensemble. The red solid line corresponds to the minimum temperature of space time and the red dashed lines in (a), (b), and (c) correspond to the locations of Hawking-Page phase transition, first-order phase transition, and second-order phase transition individually.

Substituting these critical values into (7), we can get the critical temperature:

$$
T_{c}=\frac{\sqrt{3}(3+5)}{10 l \pi} .
$$

We plot the discharge curves for different charges in Figure 1 . For the case $Q=0$, there is a minimum temperature $T_{0}=\sqrt{2} / \pi$ [30], which is indicated by the red solid line in (a). When the temperature is lower than $T_{0}$, we have only a thermal AdS. When the temperature is higher than $T_{0}$, there are two additional black hole branches. The small branch is unstable while the large branch is stable. This can be justified by checking the corresponding heat capacities, which is related to their slopes. The Hawking-Page phase transition occurs at the temperature given by $T_{1}=3 / 2 \pi$ [30], which is higher than $T_{0}$ and indicated by the red dashed line. This can be observed by the $F-T$ relation in Figure 2(a), where $T_{0}$ is the horizontal coordinate of the cusp and $T_{1}$ is the horizontal coordinate for the intersection of the stable branch and the horizontal axis. Obviously, when the temperature is lower than $T_{1}$, the thermal AdS is the most stable state. While when the temperature is higher than $T_{1}$, the most stable state is taken over by the large black hole branch.
For the case $Q \neq 0$, the phase structure is similar to that of the van der Waals phase transition. That is, for a small charge, there is an unstable black hole interpolating between the stable small hole and stable large hole. The small stable hole will jump to the large stable hole at the critical temperature $T_{\star}$, which is labeled by the red dashed line in Figure 1(b). As the charge increases to the critical charge, the small hole and the large hole merge into one and squeeze out the unstable phase. So there is an inflection point in Figure 1(c). The heat capacity is divergent in this case; the phase transition is therefore second order. As the charge exceeds the critical charge, we simply have one stable black hole at each temperature, which can be justified by the slope of the curve in Figure 1(d). The van der Waalslike phase transition can also be observed from the $F-T$ relation. From Figure 2(b), we see a swallowtail structure, which corresponds to the unstable phase in Figure 1(b). The critical temperature $T_{\star}=0.4526$ for the phase transition is apparently read off by the horizontal coordinate of the junction between the small black hole and the large black hole. As the temperature is lower than the critical temperature $T_{\star}$, the free energy of the small black hole is lowest, so the small hole is stable. As the temperature is higher than $T_{\star}$, 


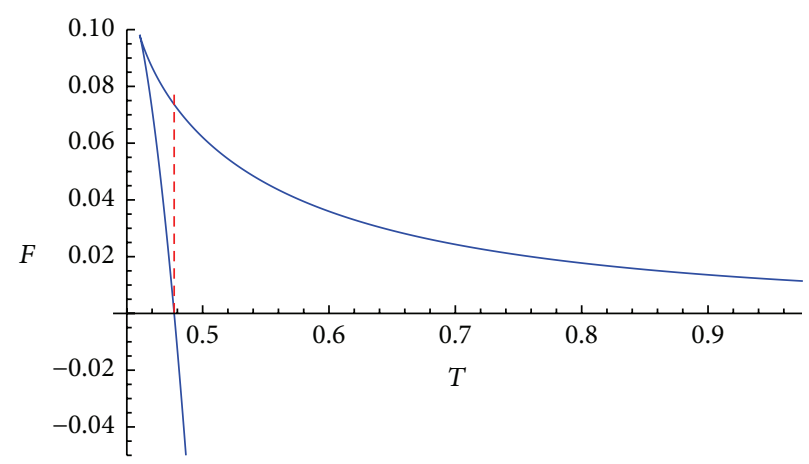

(a) $Q=0$

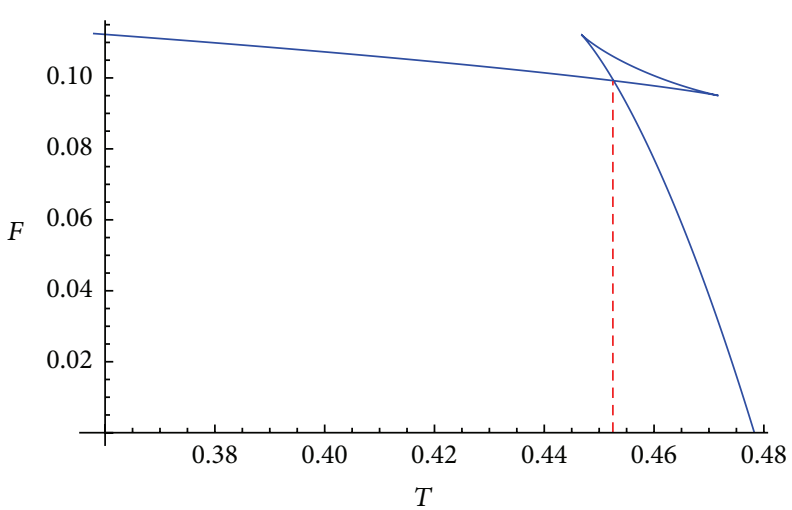

(b) $\mathrm{Q}=(\pi-1) / 6 \sqrt{5}$

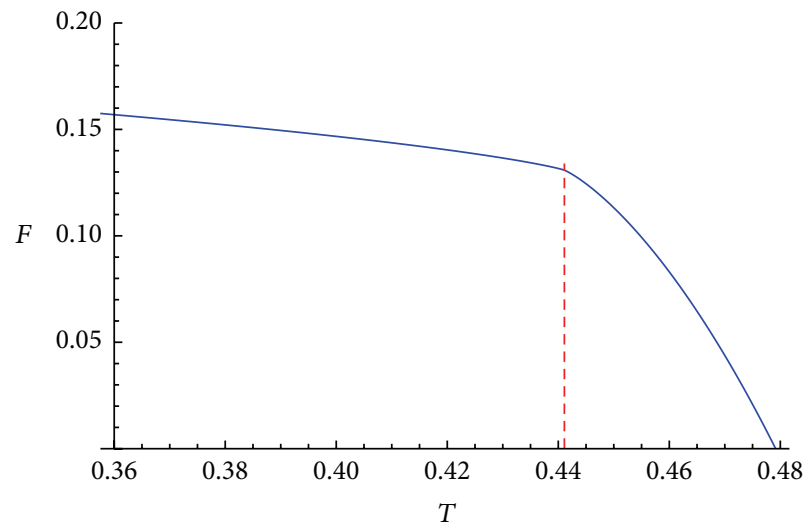

(c) $Q=\pi / 6 \sqrt{5}$

FiguRE 2: Relation between the free energy and temperature for different charges. The horizontal coordinates of the red dashed lines correspond to the temperatures of the Hawking-Page phase transition, first-order phase transition, and second-order phase transition.

the free energy of the large black hole is lowest, so the large hole dominates thereafter. The nonsmoothness of the junction indicates that the phase transition is first order. When the charge is arriving at the critical charge $Q_{c}$, the swallowtail structure in Figure 2(b) shrinks into a point as is shown in Figure 2(c). The horizontal coordinate of the inflection point corresponds to the critical temperature $T_{c}$ of the second-order phase transition, which is consistent with the analytical result in (11).

For the first-order phase transition in Figure 1(b), we would like to check whether Maxwell's equal area law holds with the following formula:

$$
A_{1} \equiv \int_{S_{1}}^{S_{3}} T(S, Q) d S=T_{\star}\left(S_{3}-S_{1}\right) \equiv A_{3}
$$

in which $T(S, Q)$ is defined in (7); $S_{1}$ and $S_{3}$ are the smallest and largest roots of the equation $T(S, Q)=T_{\star}$. After a simple calculation, we find $S_{1}=0.186987$ and $S_{3}=2.60575$. With these values, we find that $A_{1}$ and $A_{3}$ in (12) equal 1.09481 and 1.09482 , respectively. So the equal area law in the $T-S$ plane holds within our numerical accuracy.
For the second-order phase transition in Figure 1(c), we are interested in the critical exponent associated with the heat capacity:

$$
C_{Q}=\left.T \frac{\partial S}{\partial T}\right|_{Q}
$$

Near the critical point, writing the entropy as $S=S_{c}+\delta$ and expanding the temperature in terms of small $\delta$, we find

$$
\begin{aligned}
T & -T_{c} \\
& =\frac{\left(220 l^{2} \pi^{2} Q^{2}-212^{1 / 3} l^{2} \pi^{4 / 3} S^{4 / 3}+30 S^{2}\right)}{2432^{2 / 3} l^{2} \pi^{5 / 3} S^{14 / 3}}\left(S-S_{c}\right)^{3},
\end{aligned}
$$

in which we have used (9). In this case, (13) further implies $C_{\mathrm{Q}} \sim\left(T-T_{c}\right)^{-2 / 3}$; namely, the critical exponent is $-2 / 3$, which is the same as the one from the mean field theory. In addition, taking logarithm to (14), we have a linear relation:

$$
\log \left|T-T_{c}\right|=3 \log \left|S-S_{c}\right|+\text { constant }
$$

with 3 the slope. In what follows, we will use this logarithm to check the critical exponent for the analogous heat capacities in the framework of holography. 
It is noteworthy that by holography the whole phase structure described above is not only for the bulk black hole but also for the dual boundary system, where the thermal entropy is simply given by the black hole entropy, and so on.

\section{Phase Transition in the Framework of Holography}

In this section we shall investigate the phase structures of some nonlocal observables such as two-point correlation function, Wilson loop, and entanglement entropy in the dual fled theory by holography to see whether they have the same phase structure as the thermal entropy.

3.1. Phase Transition of Two-Point Correlation Function. According to the AdS/CFT correspondence, if the conformal dimension $\Delta$ of scalar operator $\mathcal{O}$ of dual field theory is large enough, the equal time two-point correlation function can be holographically approximated as [31]

$$
\left\langle\mathcal{O}\left(t_{0}, x_{i}\right) \mathcal{O}\left(t_{0}, x_{j}\right)\right\rangle \approx e^{-\Delta L}
$$

where $L$ is the length of the bulk geodesic between the points $\left(t_{0}, x_{i}\right)$ and $\left(t_{0}, x_{j}\right)$ on the AdS boundary. Taking into account the spherical symmetry of the 5-dimensional Reissner-Nordström-AdS black hole, we can simply choose $\left(\phi=\pi / 2, \theta=\theta_{0}, \psi=0\right)$ and $\left(\phi=\pi / 2, \theta=\theta_{0}, \psi=\pi\right)$ as the two boundary points. Then with $\theta$ to parameterize the trajectory, the proper length is given by

$$
\begin{aligned}
L & =2 \int_{0}^{\theta_{0}} \mathscr{L}(r(\theta), \theta) d \theta, \\
\mathscr{L} & =\sqrt{\frac{\dot{r}^{2}}{f(r)}+r^{2}},
\end{aligned}
$$

in which $\dot{r}=d r / d \theta$. Imagining $\theta$ as time, and treating $\mathscr{L}$ as the Lagrangian, one can get the equation of motion for $r(\theta)$ by making use of the Euler-Lagrange equation; that is

$$
0=\dot{r}^{2} f^{\prime}(r)-2 f(r) \ddot{r}+2 r f(r)^{2},
$$

which can be solved by imposing the following boundary conditions:

$$
\begin{aligned}
& \dot{r}(0)=0, \\
& r(0)=r_{0} .
\end{aligned}
$$

To explore whether the size of the boundary region affects the later phase structure, we here choose $\theta_{0}=0.14,0.2$ as two examples. Note that, for a fixed $\theta_{0}$, the geodesic length is divergent, so it should be regularized by subtracting off the geodesic length in pure AdS with the same boundary region, denoted by $L_{0}$. To achieve this, we are required to set a UV cutoff for each case, which is chosen to be $r(0.139)$ and $r(0.199)$, respectively, for our two examples. In this paper, we obtain $L_{0}$ also by numerics though there is an analytical result for $r\left(\theta_{0}\right)$ for pure AdS in Einstein gravity. We label the regularized geodesic length as $\delta L \equiv L-L_{0}$.
We plot the relation between $T$ and $\delta L$ for different $\theta_{0}$ in Figures 3 and 4. As shown in Figures 3 and 4, $\delta L$ demonstrates a similar phase structure as the thermal entropy. Moreover, we find that the minimum temperature $T_{0}$ as well as Hawking-Page phase transition temperature $T_{1}$ in (a), the first-order phase transition temperature $T_{\star}$ in (b), and second-order phase transition temperature $T_{c}$ in (c) are exactly the same as those in $T$ - $S$ plane, which justifies our notation. To be more specific, it is easy to check $T_{0}$ by locating the position of local minimum. But in order to confirm $T_{\star}$ and $T_{c}$, we are required to examine the equal area law for the first-order phase transition and obtain $-2 / 3$ as the critical exponent for the second-order phase transition, which are documented as follows.

In the $\delta L-T$ plane, we define the equal area law as

$$
A_{1} \equiv \int_{\delta L_{1}}^{\delta L_{3}} T(\delta L) d \delta L=T_{\star}\left(\delta L_{3}-\delta L_{1}\right) \equiv A_{3},
$$

in which $T(\delta L)$ is an Interpolating Function obtained from the numeric result and $\delta L_{1}$ and $\delta L_{3}$ are the smallest and largest roots of the equation $T(\delta L)=T_{\star}$. For the case $\theta_{0}=$ 0.14 , we find $\delta L_{1}=0.0000556147, \delta L_{3}=0.000194497$. Substituting these values into (20), we find $A_{1}=0.0000628401$, $A_{3}=0.0000628583$. For the case $\theta_{0}=0.2$, after simple calculation, we find $A_{1}=0.0000108924, A_{3}=0.0000108875$. It is obvious that for different $\theta_{0}, A_{1}$, and $A_{3}$ are equal within our numeric accuracy. Thus, the equal area law also holds in the $\delta L-T$ plane.

In addition, in order to investigate the critical exponent for the analogous heat capacity of the geodesic length. We are interested in the logarithm of the quantities $T-T_{c}, \delta L-\delta L_{c}$, in which $T_{c}$ is the critical temperature defined in (11), and $L_{c}$ is obtained numerically by the equation $T(\delta L)=T_{c}$. We plot the relation between $\log \left|T-T_{c}\right|$ and $\log \left|\delta L-\delta L_{c}\right|$ for different $\theta_{0}$ in Figure 5, where these straight lines can be fitted as

$$
\begin{aligned}
& \log \left|T-T_{c}\right| \\
& = \begin{cases}23.2318+3.06832 \log \left|\delta L-\delta L_{c}\right|, & \text { for } \theta_{0}=0.14, \\
31.9841+3.00077 \log \left|\delta L-\delta L_{c}\right|, & \text { for } \theta_{0}=0.2 .\end{cases}
\end{aligned}
$$

It is obvious that the slope is about 3 , which indicates that the critical exponent is $-2 / 3$ for the analogous heat capacity and the phase transition is also second order at $T_{c}$ for the geodesic length.

3.2. Phase Transition of Wilson Loop. In this subsection, we are going to study the phase structure of the Wilson loop, which in the bulk corresponds to the minimal area surface by holography. Wilson loop operator is defined as a path ordered integral of gauge field over a closed contour, and its expectation value is approximated geometrically by the AdS/CFT correspondence as [32]

$$
\langle W(C)\rangle \approx e^{-A_{\Sigma} / 2 \pi \alpha^{\prime}},
$$




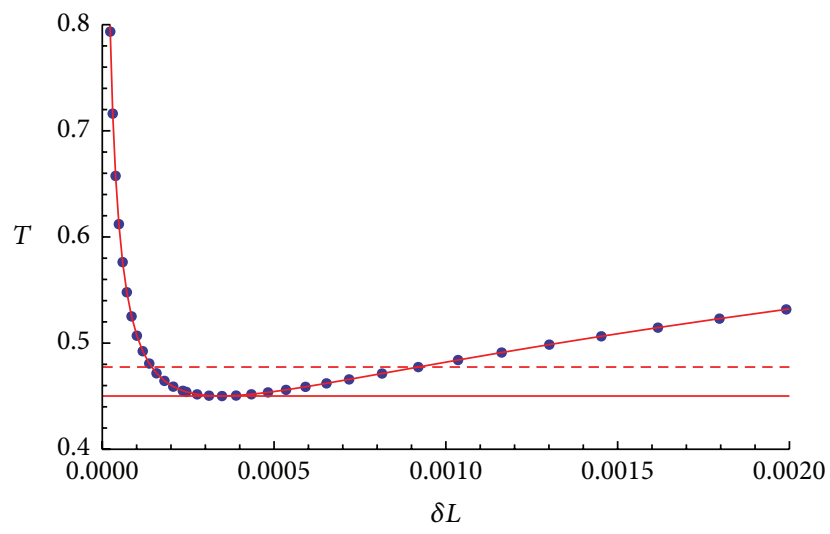

(a) $Q=0$

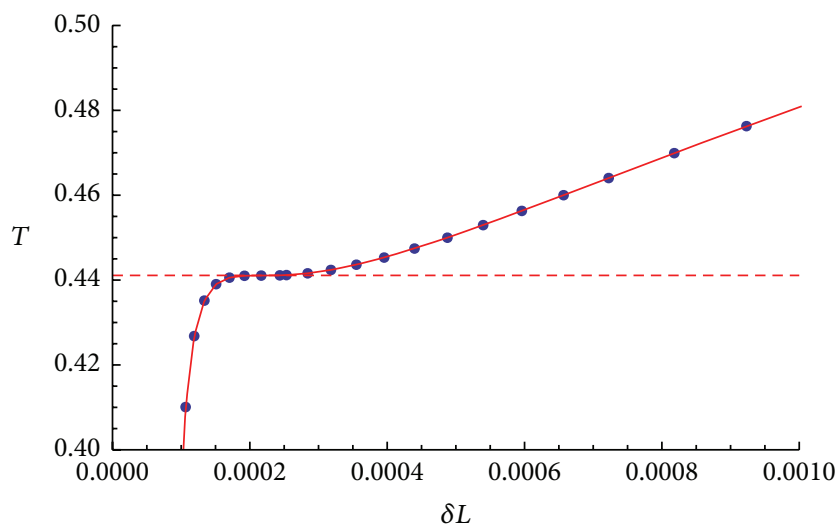

(c) $Q=\pi / 6 \sqrt{5}$

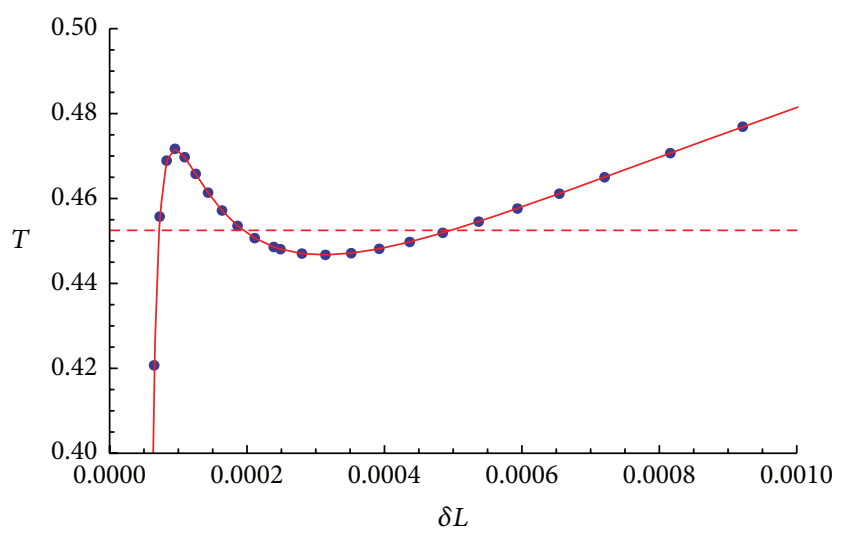

(b) $Q=(\pi-1) / 6 \sqrt{5}$

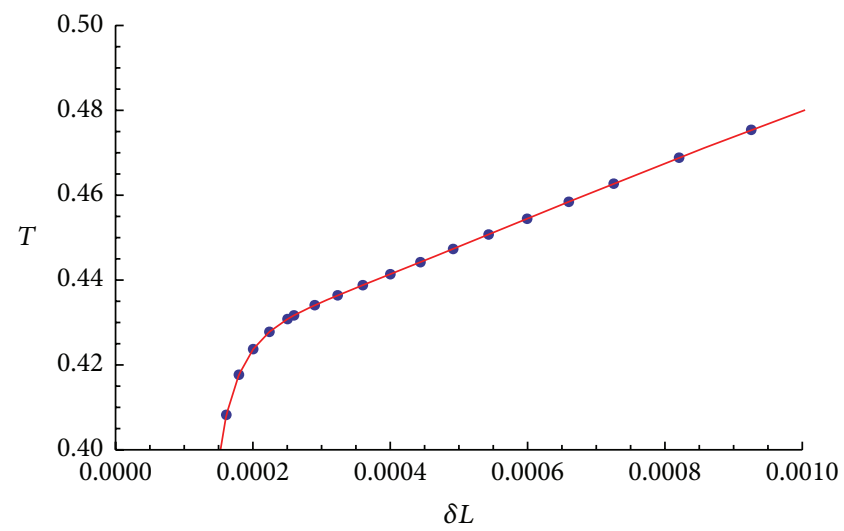

(d) $Q=(\pi+1) / 6 \sqrt{5}$

FIGURE 3: Relation between the geodesic length and temperature in the fixed charge ensemble for different charges at $\theta_{0}=0.14$. The red solid line corresponds to the location of the minimum temperature $T_{0}$; the dashed lines in (a), (b), and (c) correspond individually to the locations of Hawking-Page phase transition $T_{1}$, first-order phase transition $T_{\star}$, and second-order phase transition $T_{c}$.

where $C$ is the closed contour, $\Sigma$ is the minimal bulk surface ending on $C$ with $A$ its minimal area, and $\alpha^{\prime}$ is the Regge slope parameter. Next we choose the line with $\phi=\pi / 2$ and $\theta=\theta_{0}$ as our loop. Then we can employ $(\theta, \psi)$ to parameterize the minimal area surface, which is invariant under the $\psi$-direction by our rotational symmetry. Thus, the corresponding minimal area surface can be expressed as

$$
A=2 \pi \int_{0}^{\theta_{0}} r \sin \theta \sqrt{\frac{\dot{r}^{2}}{f(r)}+r^{2}} d \theta
$$

in which $\dot{r}=d r / d \theta$. Making use of the Euler-Lagrange equation, one can get the equation of motion for $r(\theta)$. Then with the boundary conditions $r^{\prime}(0)=0, r(0)=r_{0}$, we can further get the numeric result of $r(\theta)$. Similar to the case of geodesic length, we choose $\theta_{0}=0.14,0.2$ as two examples and the corresponding UV cutoffs are set to be $r(0.139)$, $r(0.199)$. We label the regularized minimal area surface as $\delta A \equiv A-A_{0}$, where $A_{0}$ is the minimal area in pure AdS with the same boundary region. We plot the relation between $\delta A$ and $T$ for different $\theta_{0}$ in Figures 6 and 7. Comparing Figure 6 with Figure 7, we find they are the same nearly besides the scale of the horizontal coordinate. In other words, $\theta_{0}$ affects only the value but not the phase structure of minimal area surface in the $T-\delta A$ plane. The result tells us that the similar phase structure also shows up for the minimal surface area. Here we concentrate only on scrutinizing the equal area law for the first-order phase transition and the critical exponent of the analogous heat capacity for the second-order phase transition.

First, in the $\delta A-T$ plane, the equal area law can be similarly defined as

$$
A_{1} \equiv \int_{\delta A_{1}}^{\delta A_{3}} T(\delta A) d \delta A=T_{\star}\left(\delta A_{3}-\delta A_{1}\right) \equiv A_{3}
$$

in which $T(\delta A)$ is an Interpolating Function obtained from our numeric result and $\delta A_{1}$ and $\delta A_{3}$ are the smallest and largest roots of the equation $T(\delta A)=T_{\star}$, respectively. As the same as that of the geodesic length, for a fixed $\theta_{0}$, we first obtain $\delta A_{1}$ and $\delta A_{3}$ and then substitute these values into (24) to produce $A_{1}, A_{3}$. The concrete values are listed in Table 1. Obviously, for both $\theta_{0}, A_{1}$ and $A_{3}$ are equal within the reasonable numeric accuracy. The equal area law thus holds in the $\delta A-T$ plane, which reinforces the fact that the 


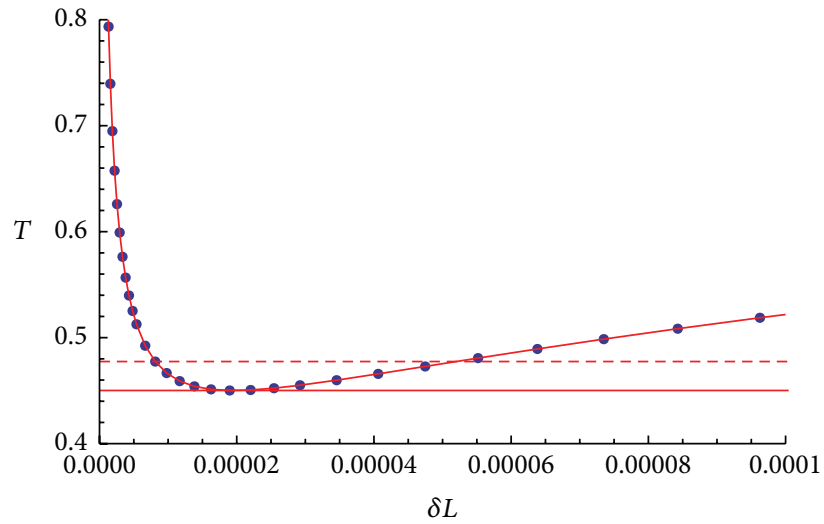

(a) $Q=0$

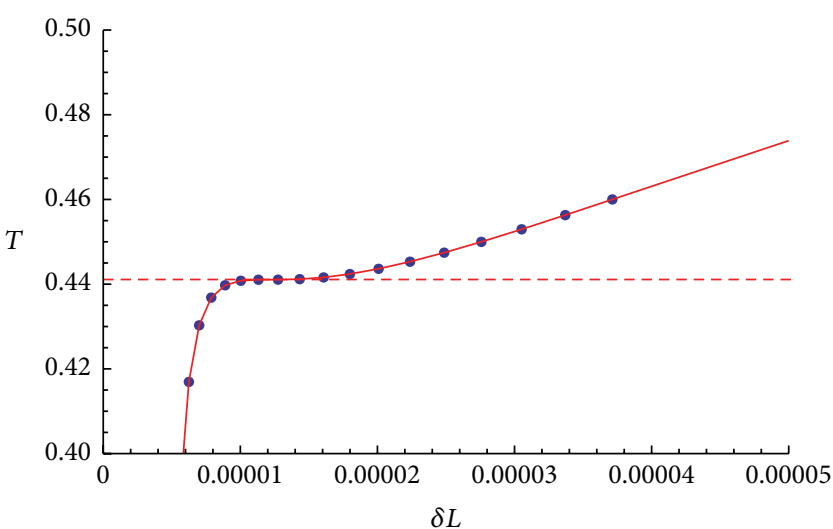

(c) $Q=\pi / 6 \sqrt{5}$

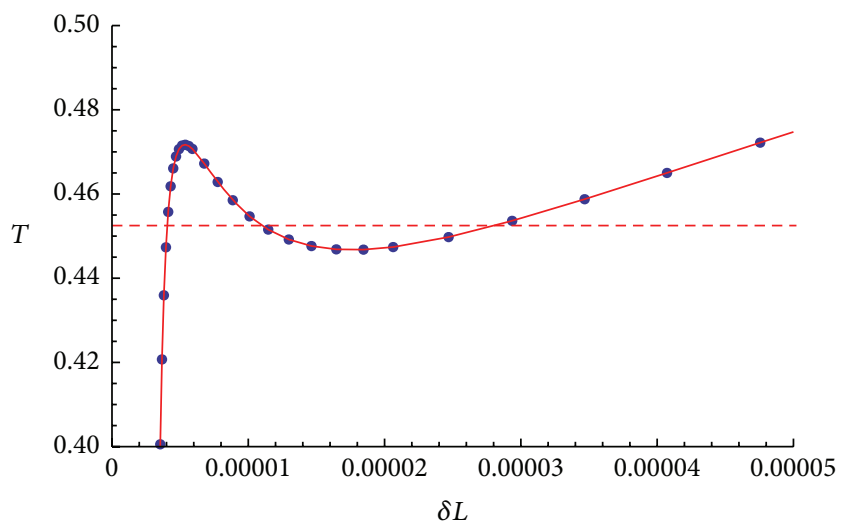

(b) $Q=(\pi-1) / 6 \sqrt{5}$

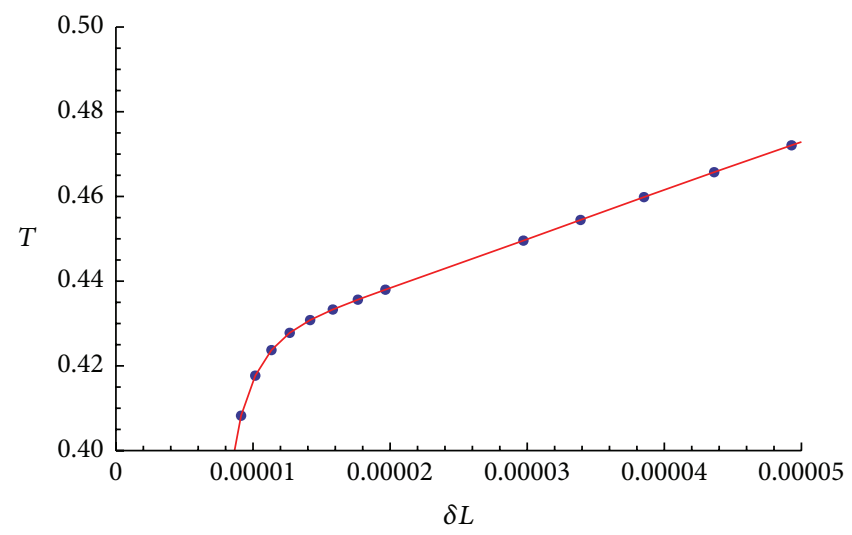

(d) $Q=(\pi+1) / 6 \sqrt{5}$

FIGURE 4: Relation between the geodesic length and temperature in the fixed charge ensemble for different charges at $\theta_{0}=0.2$. The red solid line corresponds to the location of the minimum temperature $T_{0}$; the dashed lines in (a), (b), and (c) correspond individually to the locations of Hawking-Page phase transition $T_{1}$, first-order phase transition $T_{\star}$, and second-order phase transition $T_{c}$.

minimal surface area has the same first-order phase transition behavior as that of the thermal entropy.

Second, in order to check whether the minimal surface area also demonstrates the same second-order phase transition as the thermal entropy, we would like to evaluate the critical exponent of the analogous heat capacity at the critical point in the $\delta A-T$ plane. To this end, we plot the relations between $\log \left|T-T_{c}\right|$ and $\log \left|\delta A-\delta A_{c}\right|$ in Figure 8. The numerical results for these curves can be fitted as

$$
\begin{aligned}
& \log \left|T-T_{c}\right| \\
& = \begin{cases}27.5226+3.04698 \log \left|\delta A-\delta A_{c}\right|, & \text { for } \theta_{0}=0.14, \\
24.692+3.00462 \log \left|\delta A-\delta A_{c}\right|, & \text { for } \theta_{0}=0.2 .\end{cases}
\end{aligned}
$$

With 3 the slope, we can conclude that the minimal surface area also has the same second-order phase transition as the thermal entropy.

3.3. Phase Transition of Entanglement Entropy. Holographic entanglement entropy is another nonlocal observable, and it has been used extensively to probe the superconductivity phase transition besides the thermalization process recently [33-40]. In this subsection, we intend to employ it to probe the phase structure of a 5-dimensional Reissner-NordströmAdS black hole. According to the formula in [41, 42], holographic entanglement entropy can be given by the area $A_{\Sigma}$ of a minimal surface $\Sigma$ anchored on the boundary entangling surface $\partial \Sigma$; namely,

$$
S=\frac{A_{\Sigma}(t)}{4 G}
$$

For simplicity, we choose $\phi=\phi_{0}$ as our entangling surface and employ $(\phi, \theta, \psi)$ to parameterize the minimal surface. But with the symmetry of (2), (26) can be rewritten as

$$
S=4 \pi \int_{0}^{\phi_{0}} r^{2} \sin ^{2} \phi \sqrt{\frac{\dot{r}^{2}}{f(r)}+r^{2}} d \phi
$$

with $\dot{r}=d r / d \phi$. Similarly, we can solve the equation of motion for $r(\phi)$ numerically and eventually obtain the regularized entanglement entropy $\delta S$. We plot the relation 


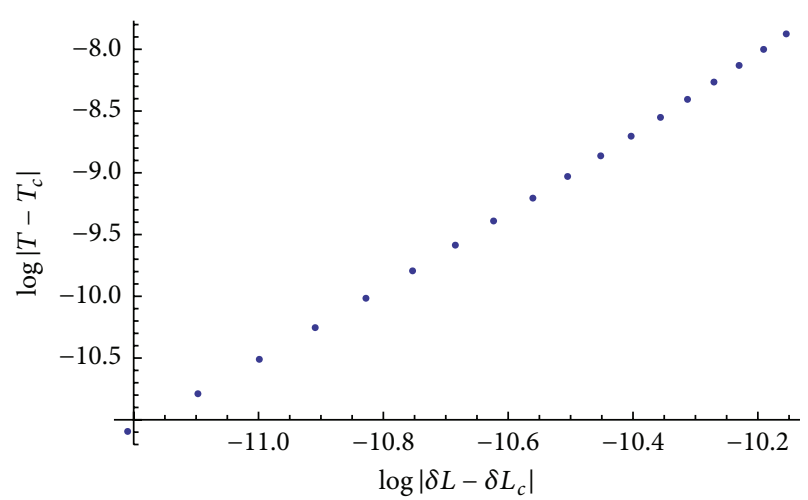

(a) $\theta_{0}=0.14$

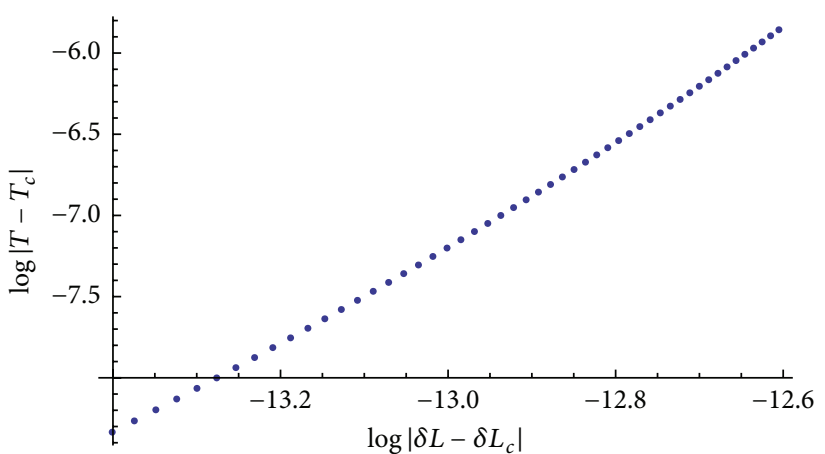

(b) $\theta_{0}=0.2$

FIgURE 5: Relation between $\log \left|T-T_{c}\right|$ and $\log \left|\delta L-\delta L_{c}\right|$ near the critical point of second-order phase transition for different $\theta_{0}$.

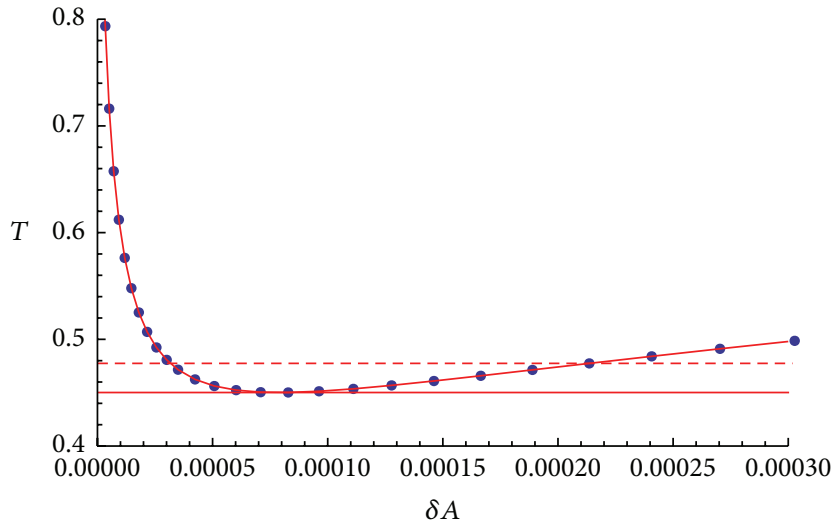

(a) $Q=0$

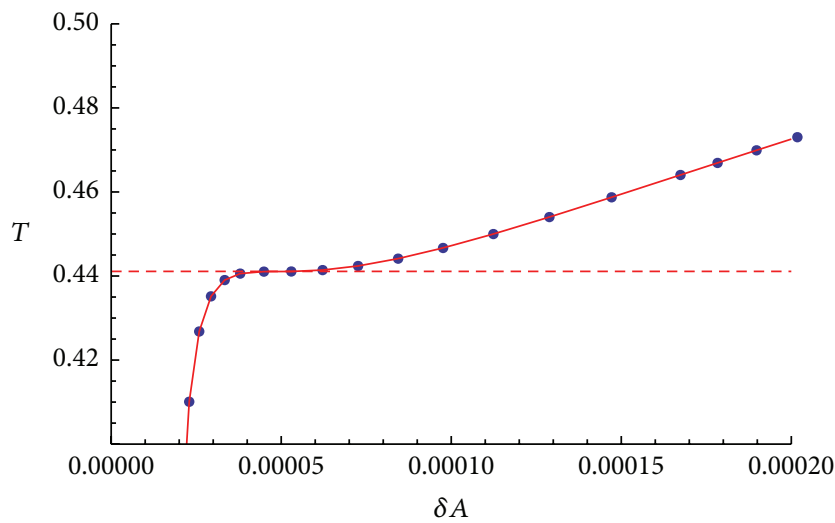

(c) $Q=\pi / 6 \sqrt{5}$

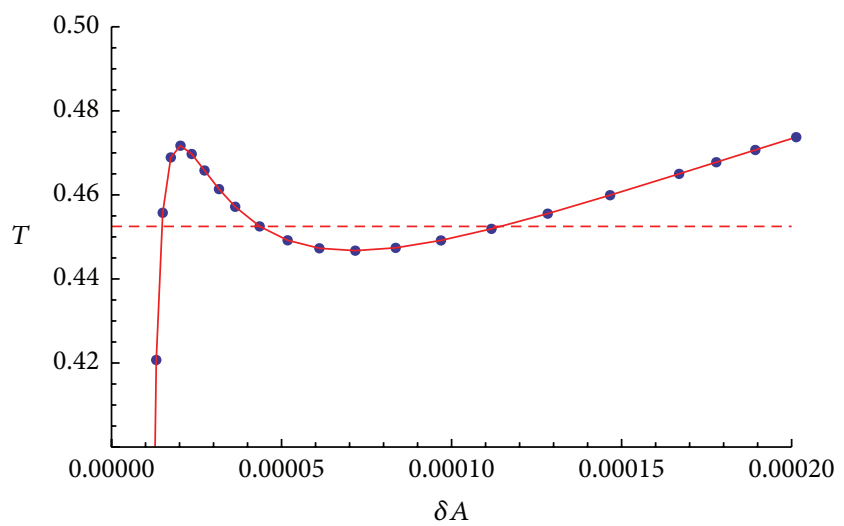

(b) $Q=(\pi-1) / 6 \sqrt{5}$

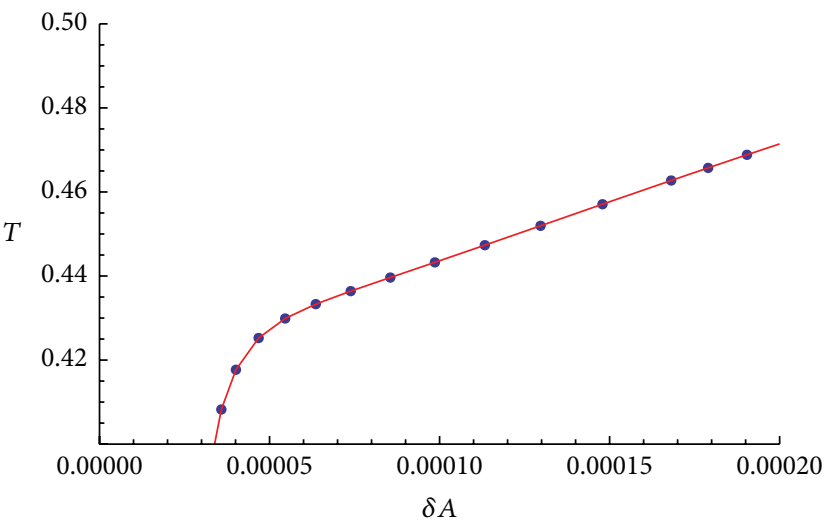

(d) $Q=(\pi+1) / 6 \sqrt{5}$

FiguRE 6: Relation between the minimal area surface and temperature in the fixed charge ensemble for different charges at $\theta_{0}=0.14$. The red solid line corresponds to the location of the minimum temperature $T_{0}$; the dashed lines in (a), (b), and (c) correspond individually to the locations of Hawking-Page phase transition $T_{1}$, first-order phase transition $T_{\star}$, and second-order phase transition $T_{c}$.

TABLE 1: Check of the equal area law in the $T-\delta A$ plane for different $\theta_{0}$.

\begin{tabular}{lc}
\hline$\theta_{0}=0.14$ & $\theta_{0}=0.2$ \\
$T_{\star}=0.4526$ & $T_{\star}=0.4526$ \\
$\delta A_{1}=0.0000434217 \mid \delta A_{3}=0.000114835$ & $\delta A_{1}=0.0000374703 \mid \delta A_{3}=0.000248923$ \\
$A_{1}=0.0000320531 \mid A_{3}=0.0000323218$ & $A_{1}=0.0000957474 \mid A_{3}=0.0000957035$
\end{tabular}




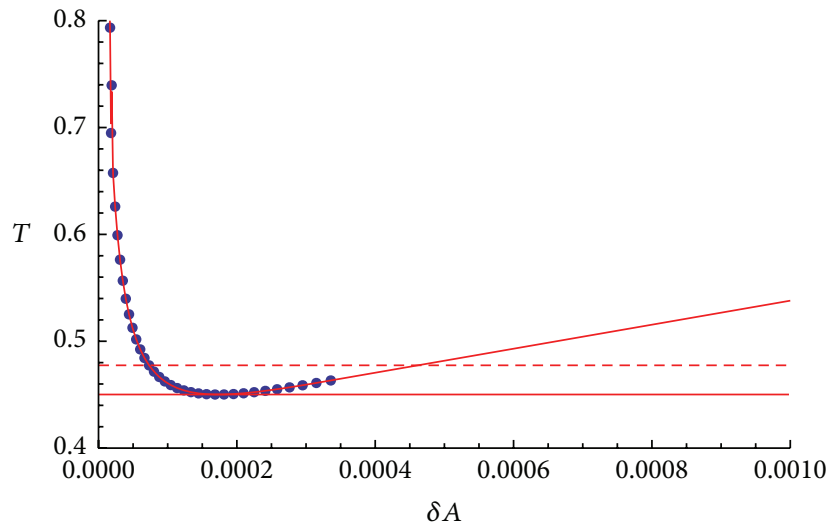

(a) $Q=0$

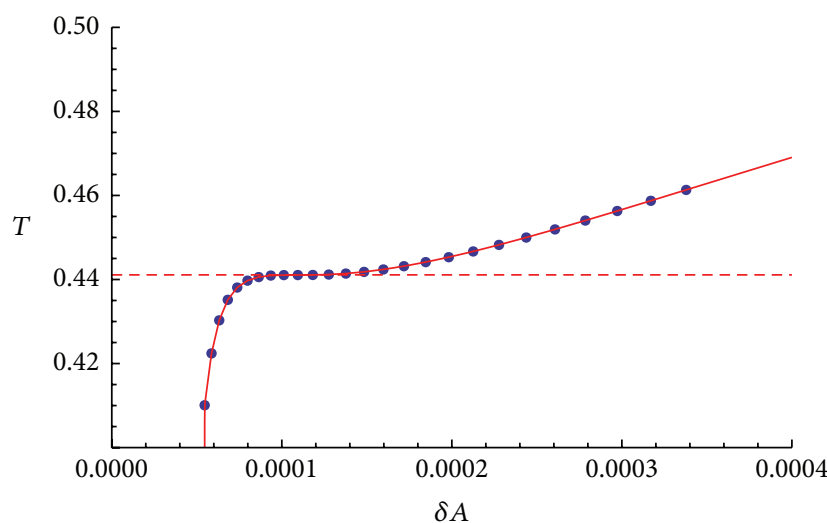

(c) $Q=\pi / 6 \sqrt{5}$

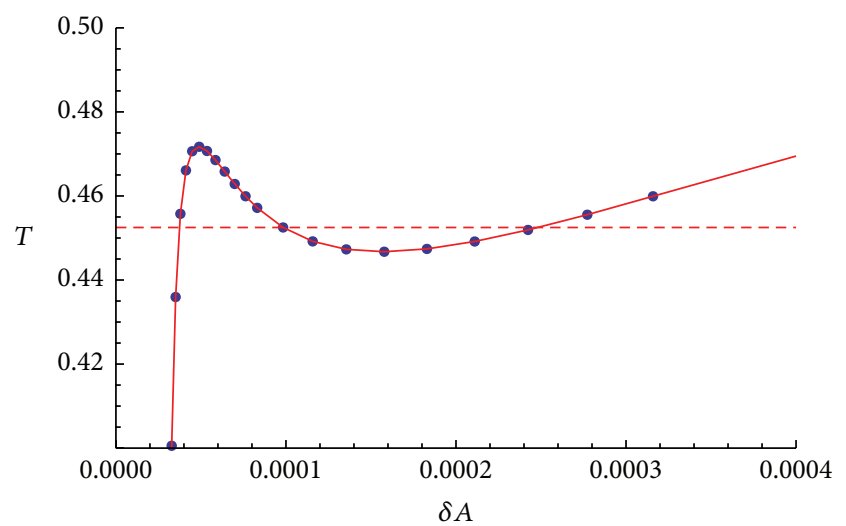

(b) $\mathrm{Q}=(\pi-1) / 6 \sqrt{5}$

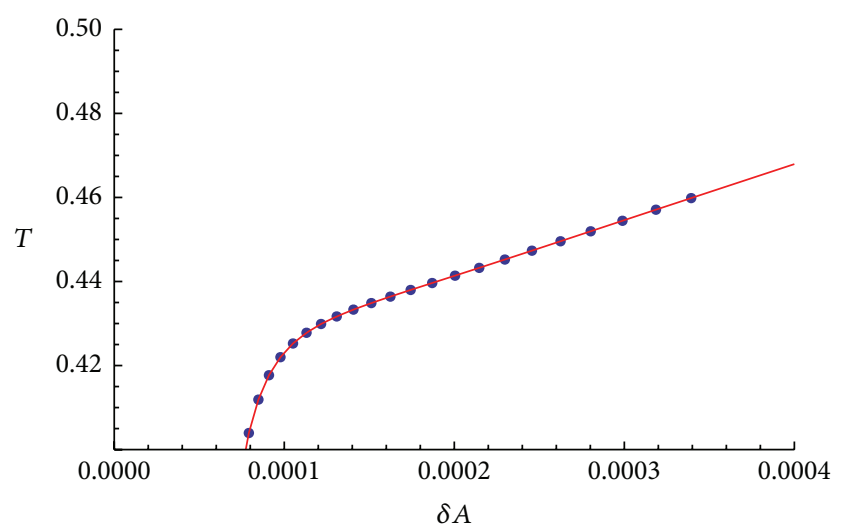

(d) $Q=(\pi+1) / 6 \sqrt{5}$

FiguRE 7: Relation between the minimal area surface and temperature in the fixed charge ensemble for different charges at $\theta_{0}=0.2$. The red solid line corresponds to the location of the minimum temperature $T_{0}$; the dashed lines in (a), (b), and (c) correspond individually to the locations of Hawking-Page phase transition $T_{1}$, first-order phase transition $T_{\star}$, and second-order phase transition $T_{c}$.

between $\delta S$ and $T$ for $\phi_{0}=0.14,0.2$ in Figures 9 and 10, respectively. As one can see, it exhibits a similar behavior as the thermal entropy. To be more precise, we would like to check the equal area law with the following equation:

$$
A_{1} \equiv \int_{\delta S_{1}}^{\delta S_{3}} T(\delta S) d \delta S=T_{\star}\left(\delta S_{3}-\delta S_{1}\right) \equiv A_{3}
$$

in which $T(\delta S)$ is an Interpolating Function obtained from the numeric result and $\delta S_{1}$ and $\delta S_{3}$ are the smallest and largest roots of the equation $T(\delta S)=T_{\star}$. For different $\phi_{0} s$, the results of $\delta S_{1}, \delta S_{3}$ and $A_{1}, A_{3}$ are listed in Table 2. It is obvious that $A_{1}$ nearly equals $A_{3}$ regardless of the choice of $\phi_{0}$. That is, the equal area law is also valid for the entanglement entropy.

To get the critical exponent of second-order phase transition of entanglement entropy, we should find the slope of a linear function represented by $\log \left|T-T_{c}\right|$ and $\log \left|\delta S-\delta S_{c}\right|$, in which $S_{c}$ is the critical entropy obtained numerically by the equation $T(\delta S)=T_{c}$. The numeric results for different $\phi_{0}$ are plotted in Figure 11. The results for these curves can be further fitted as

$$
\begin{aligned}
& \log \left|T-T_{c}\right| \\
& = \begin{cases}26.653+3.00107 \log \left|\delta S-\delta S_{c}\right|, & \text { for } \phi_{0}=0.14 \\
21.2674+2.92789 \log \left|\delta S-\delta S_{c}\right|, & \text { for } \phi_{0}=0.2 .\end{cases}
\end{aligned}
$$

One can see that the slope is always about 3 for different $\phi_{0}$. So we can conclude that the entanglement entropy also has the same second-order phase transition as the thermal entropy.

\section{Concluding Remarks}

Investigation on the phase transition of the black holes is important and necessary. On the one hand, it is helpful for us to understand the structure and nature of the space time. On the other hand, it may uncover some phase transitions of the realistic physics in the conformal field theory according to the AdS/CFT correspondence. It is well known now that the Hawking-Page phase transition in the gravity system 


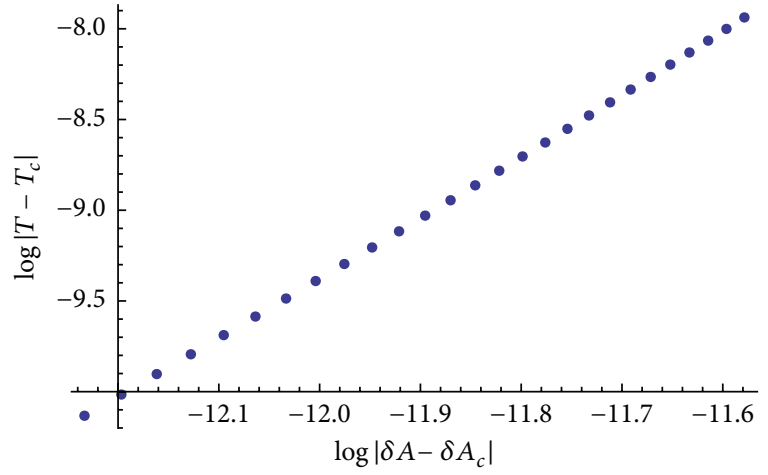

(a) $\theta_{0}=0.14$

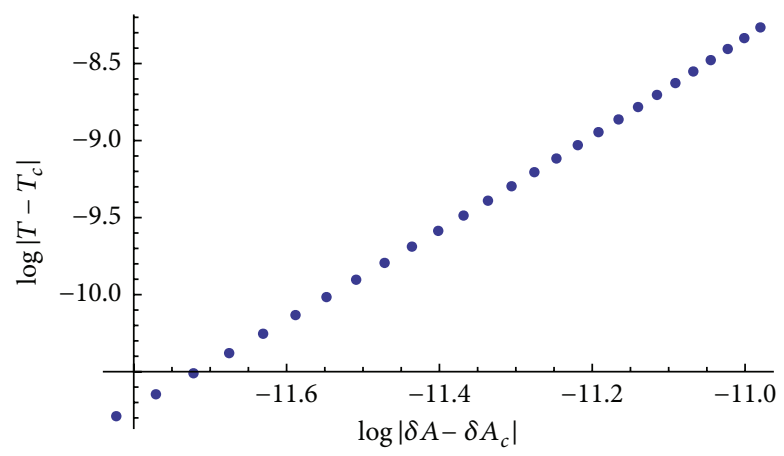

(b) $\theta_{0}=0.2$

Figure 8: Relation between $\log \left|T-T_{c}\right|$ and $\log \left|\delta A-\delta A_{c}\right|$ near the critical point of second-order phase transition for different $\theta_{0}$.

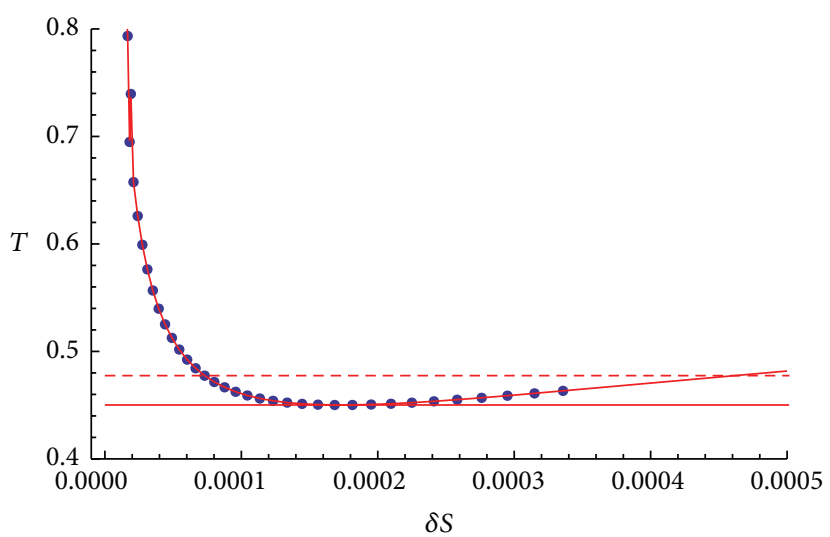

(a) $Q=0$

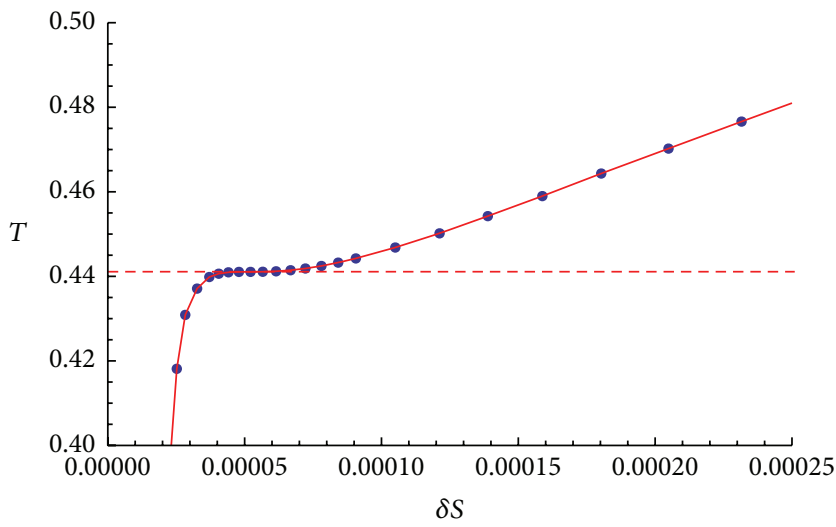

(c) $Q=\pi / 6 \sqrt{5}$

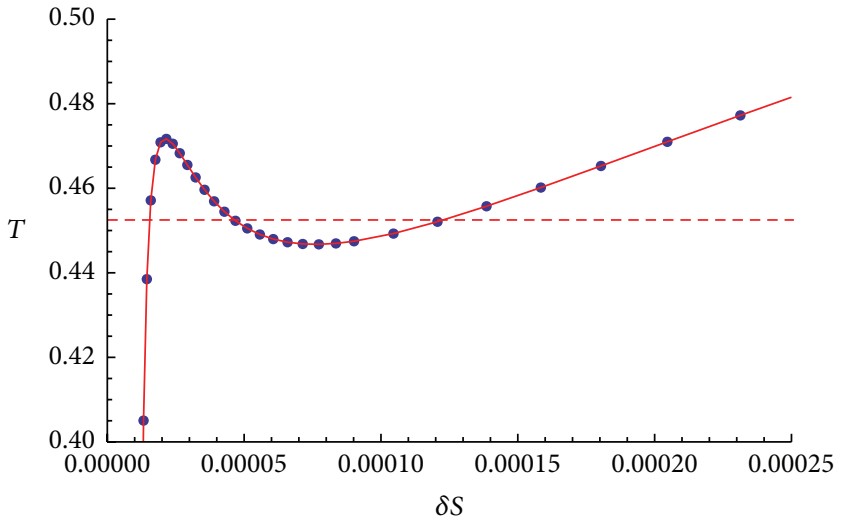

(b) $Q=(\pi-1) / 6 \sqrt{5}$

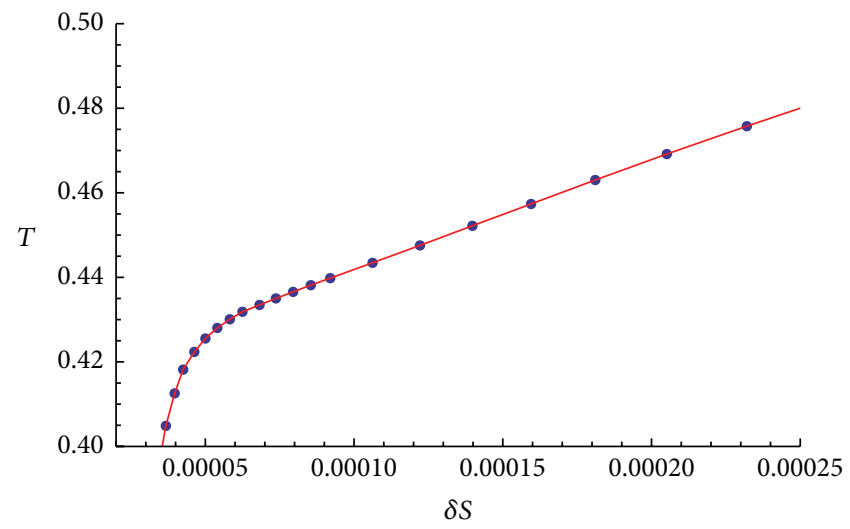

(d) $Q=(\pi+1) / 6 \sqrt{5}$

Figure 9: Relation between the entanglement entropy and temperature in the fixed charge ensemble for different charges at $\phi_{0}=0.14$. The red solid line corresponds to the location of the minimum temperature $T_{0}$; the dashed lines in (a), (b), and (c) correspond individually to the locations of Hawking-Page phase transition $T_{1}$, first-order phase transition $T_{\star}$, and second-order phase transition $T_{c}$.

TABLE 2: Check of the equal area law in the $T-\delta S$ plane for different $\phi_{0}$.

\begin{tabular}{lc}
\hline$\phi_{0}=0.14$ & $\phi_{0}=0.2$ \\
$T_{\star}=0.4526$ & $T_{\star}=0.4526$ \\
$\delta S_{1}=0.0000155355 \mid \delta S_{3}=0.000123195$ & $\delta S_{1}=0.000186918 \mid \delta S_{3}=0.000517552$ \\
$A_{1}=0.0000487431 \mid A_{3}=0.0000487266$ & $A_{1}=0.000148384 \mid A_{3}=0.000149645$ \\
\hline
\end{tabular}




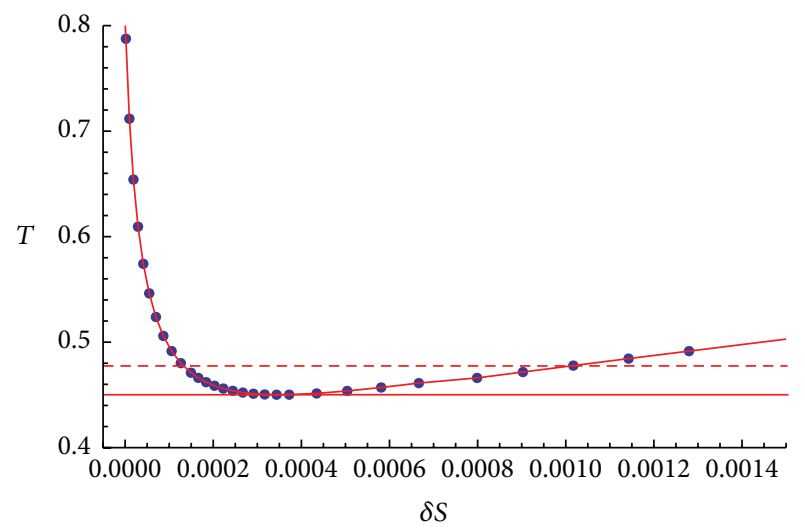

(a) $Q=0$

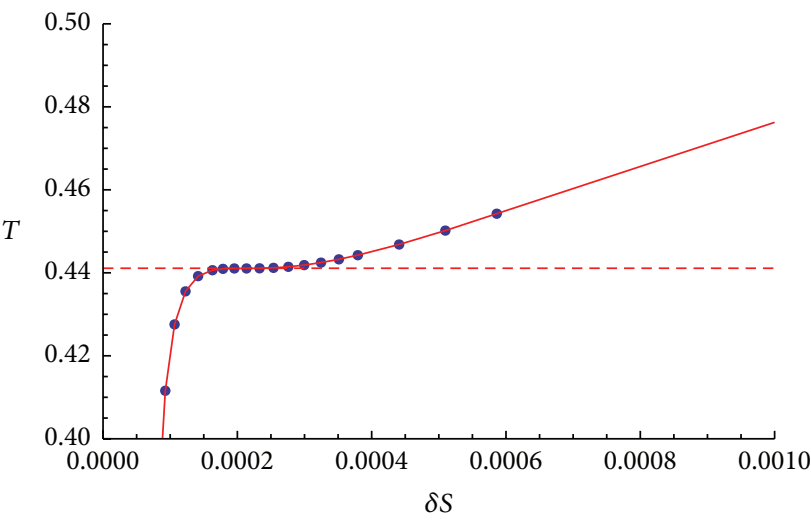

(c) $Q=\pi / 6 \sqrt{5}$

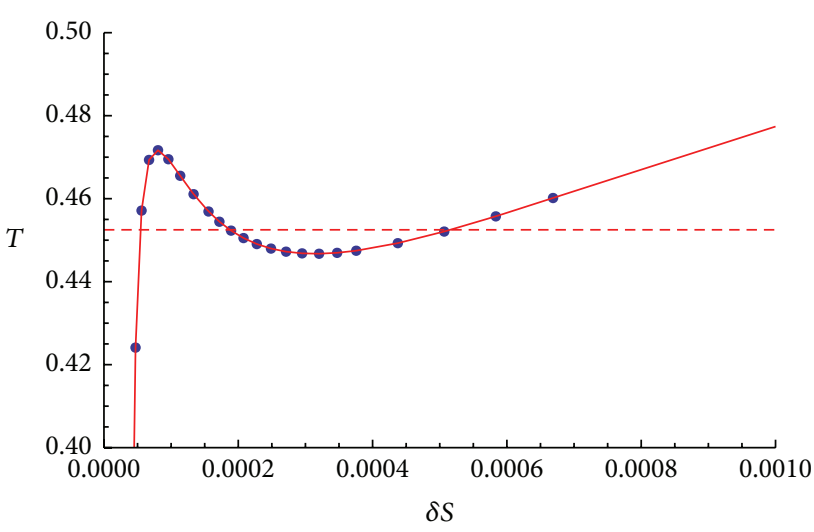

(b) $\mathrm{Q}=(\pi-1) / 6 \sqrt{5}$

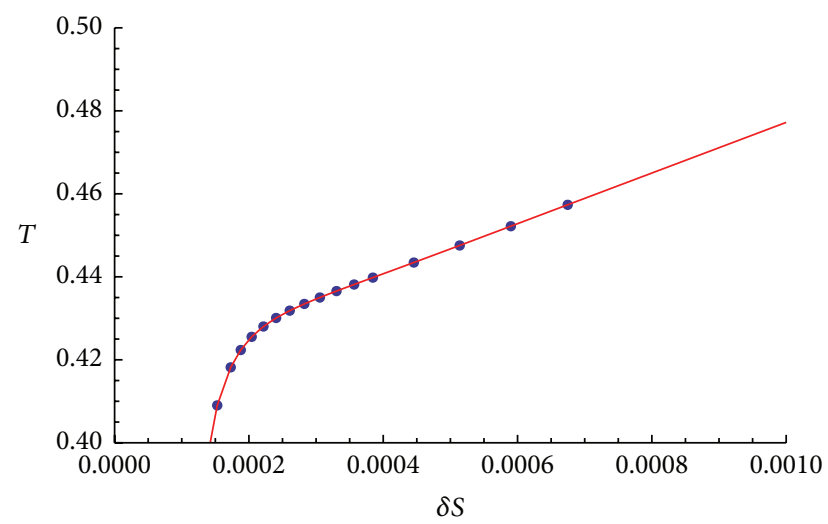

(d) $Q=(\pi+1) / 6 \sqrt{5}$

FiguRE 10: Relation between the entanglement entropy and temperature in the fixed charge ensemble for different charges at $\phi_{0}=0.2$. The red solid line corresponds to the location of the minimum temperature $T_{0}$; the dashed lines in (a), (b), and (c) correspond individually to the locations of Hawking-Page phase transition $T_{1}$, first-order phase transition $T_{\star}$, and second-order phase transition $T_{c}$.

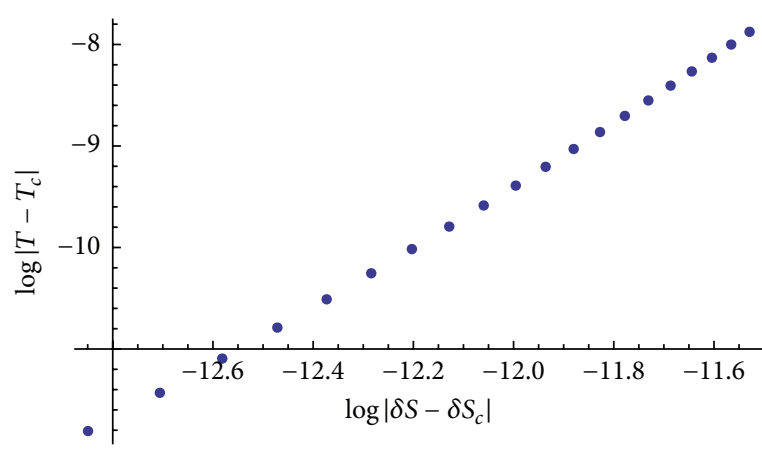

(a)

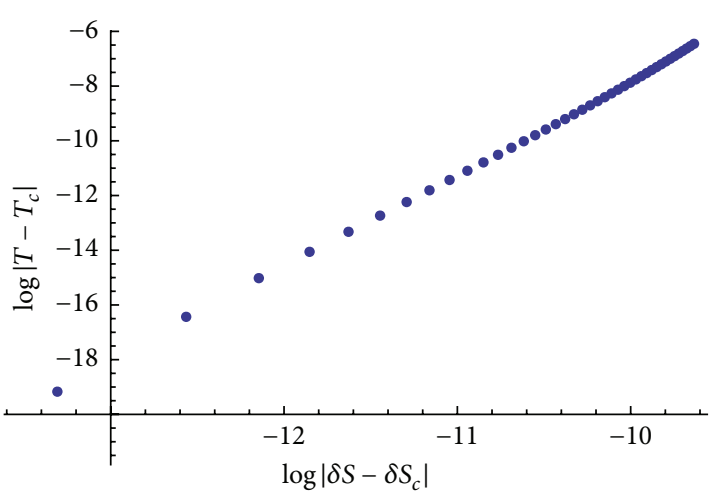

(b)

FIGURE 11: Relation between $\log \left|T-T_{c}\right|$ and $\log \left|\delta S-\delta S_{c}\right|$ near the critical point of second-order phase transition for different $\phi_{0}$.

is dual to the confinement/deconfinement phase transition, and the phase transition of a scalar field is dual to the superconductivity phase transition in the dual conformal field theory.

In this paper, we investigated the van der Waals-like phase transition in the framework of holography so that we can explore whether there is a realistic similar phase transition in physics. Taking the 5-dimensional Reissner-NordströmAdS black hole as the gravity background, we investigated the phase structure of the two-point correlation function, Wilson loop, and holographic entanglement entropy. For all the nonlocal observables, we observed that the black 
hole undergoes a van der Waals-like phase transition. This conclusion is reinforced by the investigation of the equal area law and critical exponent of the analogous heat capacity in which we found that the equal area law is valid always and the critical exponent of the heat capacity coincides with that of the mean field theory regardless of the size of the boundary region. In addition, we found the black hole undergoes a Hawking-Page phase transition before the van der Waalslike phase transition for all the nonlocal observables. We also obtained the minimum temperature and Hawking-Page phase transition temperature. Our investigation thus provides a complete picture depicting the phase transition of charged AdS black hole in the framework of holography.

\section{Competing Interests}

The authors declare that they have no competing interests.

\section{Acknowledgments}

The authors would like to thank Rong-Gen Cai for his discussions. This work is supported by the National Natural Science Foundation of China (Grants nos. 11405016 and 11575270), China Postdoctoral Science Foundation (Grant no. 2016M590138), Natural Science Foundation of Education Committee of Chongqing (Grant no. KJ1500530), and Basic Research Project of Science and Technology Committee of Chongqing (Grant no. cstc2016jcyja0364).

\section{References}

[1] S. W. Hawking, "Particle creation by black holes," Communications in Mathematical Physics, vol. 43, no. 3, pp. 199-220, 1975.

[2] J. M. Bardeen, B. Carter, and S. W. Hawking, "The four laws of black hole mechanics," Communications in Mathematical Physics, vol. 31, pp. 161-170, 1973.

[3] J. M. Maldacena, "Large N limit of superconformal field theories and supergravity," International Journal of Theoretical Physics, vol. 38, no. 4, pp. 1113-1133, 1999.

[4] E. Witten, "Anti de Sitter space and holography," Advances in Theoretical and Mathematical Physics, vol. 2, no. 2, pp. 253-291, 1998.

[5] S. S. Gubser, I. R. Klebanov, and A. M. Polyakov, "Gauge theory correlators from non-critical string theory," Physics Letters. B, vol. 428, no. 1-2, pp. 105-114, 1998.

[6] S. W. Hawking and D. N. Page, "Thermodynamics of black holes in anti-de Sitter space," Communications in Mathematical Physics, vol. 87, no. 4, pp. 577-588, 1983.

[7] R. G. Cai, L. M. Cao, and Y. W. Sun, "Hawking-page phase transition of black Dp-branes and R-charged black holes with an IR Cutoff,' Journal of High Energy Physics, vol. 2007, no. 11, article 039, 2007.

[8] E. Witten, "Anti-de Sitter space, thermal phase transition, and confinement in gauge theories," Advances in Theoretical and Mathematical Physics, vol. 2, no. 3, pp. 505-532, 1998.

[9] A. Chamblin, R. Emparan, C. V. Johnson, and R. C. Myers, "Charged AdS black holes and catastrophic holography," Physical Review D, vol. 60, no. 6, Article ID 064018, 1999.
[10] D. Kubiznak and R. B. Mann, "P-V criticality of charged AdS black holes," Journal of High Energy Physics, vol. 2012, no. 7, article 033, 2012.

[11] J. Xu, L. M. Cao, and Y. P. Hu, "P-V criticality in the extended phase space of black holes in massive gravity," Physical Review $D$, vol. 91, Article ID 124033, 2015.

[12] R.-G. Cai, L.-M. Cao, L. Li, and R.-Q. Yang, "P-V criticality in the extended phase space of Gauss-Bonnet black holes in AdS space," Journal of High Energy Physics, vol. 2013, article 5, 2013.

[13] S. H. Hendi, A. Sheykhi, S. Panahiyan, and B. Eslam Panah, "Phase transition and thermodynamic geometry of EinsteinMaxwell-dilaton black holes," Physical Review D, vol. 92, no. 6, Article ID 064028, 2015.

[14] R. A. Hennigar, W. G. Brenna, and R. B. Mann, " $P-v$ criticality in quasitopological gravity," Journal of High Energy Physics, vol. 2015, no. 7, article 77, pp. 1-31, 2015.

[15] S.-W. Wei and Y.-X. Liu, "Clapeyron equations and fitting formula of the coexistence curve in the extended phase space of charged AdS black holes," Physical Review D, vol. 91, no. 4, Article ID 044018, 2015.

[16] J.-X. Mo and W.-B. Liu, " $P-V$ criticality of topological black holes in Lovelock-Born-Infeld gravity," The European Physical Journal C, vol. 74, no. 4, article 2836, pp. 1-12, 2014.

[17] C. Niu, Y. Tian, and X. N. Wu, "Critical phenomena and thermodynamic geometry of Reissner-Nordström-anti-de Sitter black holes," Physical Review D, vol. 85, no. 2, Article ID 024017, 2012.

[18] R.-G. Cai, "Gauss-Bonnet black holes in AdS spaces," Physical Review. D. Third Series, vol. 65, no. 8, Article ID 084014, 2002.

[19] V. Balasubramanian, A. Bernamonti, J. de Boer et al., "Thermalization of strongly coupled field theories," Physical Review Letters, vol. 106, Article ID 191601, 2011.

[20] V. Balasubramanian, A. Bernamonti, J. de Boer et al., "Holographic thermalization," Physical Review D, vol. 84, no. 2, Article ID 026010, 2011.

[21] D. Galante and M. Schvellinger, "Thermalization with a chemical potential from AdS spaces," Journal of High Energy Physics, vol. 2012, no. 7, article 096, 2012

[22] E. Caceres and A. Kundu, "Holographic thermalization with chemical potential," Journal of High Energy Physics, vol. 2012, article 55, 2012.

[23] X.-X. Zeng and W.-B. Liu, "Holographic thermalization in Gauss-Bonnet gravity," Physics Letters B, vol. 726, no. 1-3, pp. 481-487, 2013

[24] X. X. Zeng, X. M. Liu, and B. W. Liu, "Holographic thermalization with a chemical potential in Gauss-Bonnet gravity," Journal of High Energy Physics, vol. 2014, article 31, 2014.

[25] X. X. Zeng, D. Y. Chen, and L. F. Li, "Holographic thermalization and gravitational collapse in a spacetime dominated by quintessence dark energy," Physical Review D, vol. 91, no. 4, Article ID 046005, 2015.

[26] C. V. Johnson, "Large $N$ phase transitions, finite volume, and entanglement entropy," Journal of High Energy Physics, vol. 2014, article 47, 2014.

[27] E. Caceres, P. H. Nguyen, and J. F. Pedraza, "Holographic entanglement entropy and the extended phase structure of STU black holes," http://arxiv.org/abs/1507.06069.

[28] P. H. Nguyen, "An equal area law for holographic entanglement entropy of the AdS-RN black hole," Journal of High Energy Physics, vol. 2015, article 139, 2015. 
[29] X. X. Zeng, H. Zhang, and L. F. Li, "Phase transition of holographic entanglement entropy in massive gravity," Physics Letters B, vol. 756, pp. 170-179, 2016.

[30] R. Banerjee and D. Roychowdhury, "Thermodynamics of phase transition in higher dimensional AdS black holes," Journal of High Energy Physics, vol. 2011, article 4, 2011.

[31] V. Balasubramanian and S. F. Ross, "Holographic particle detection," Physical Review D, vol. 61, no. 4, Article ID 044007, 2000.

[32] J. Maldacena, "Wilson loops in large $N$ field theories," Physical Review Letters, vol. 80, no. 22, pp. 4859-4862, 1998.

[33] T. Albash and C. V. Johnson, "Holographic studies of entanglement entropy in superconductors," Journal of High Energy Physics, vol. 2012, no. 5, article 79, pp. 1-21, 2012.

[34] R. G. Cai, S. He, L. Li, and Y. L. Zhang, "Holographic entanglement entropy in insulator/superconductor transition," Journal of High Energy Physics, vol. 2012, no. 7, article 088, 2012.

[35] R. G. Cai, L. Li, L. F. Li, and R. K. Su, "Entanglement entropy in holographic P-wave superconductor/insulator model," Journal of High Energy Physics, vol. 2013, article 63, 2013.

[36] L.-F. Li, R.-G. Cai, L. Li, and C. Shen, "Entanglement entropy in a holographic p-wave superconductor model," Nuclear Physics B, vol. 894, pp. 15-28, 2015.

[37] R. G. Cai, S. He, L. Li, and L. F. Li, "Entanglement entropy and Wilson loop in Stückelberg holographic insulator/superconductor model," Journal of High Energy Physics, vol. 2012, no. 10, article 107, 2012.

[38] X. Bai, B.-H. Lee, L. Li, J.-R. Sun, and H.-Q. Zhang, “Time evolution of entanglement entropy in quenched holographic superconductors," Journal of High Energy Physics, vol. 2015, p. 66, 2015.

[39] R. Cai, L. Li, L. Li, and R. Yang, "Introduction to holographic superconductor models," Science China Physics, Mechanics \& Astronomy, vol. 58, no. 6, pp. 1-46, 2015.

[40] Y. Ling, P. Liu, C. Niu, J. P. Wu, and Z. Y. Xian, "Holographic entanglement entropy close to quantum phase transitions," Journal of High Energy Physics, vol. 2016, article 114, 2016.

[41] S. Ryu and T. Takayanagi, "Holographic derivation of entanglement entropy from the anti-de Sitter space/conformal field theory correspondence," Physical Review Letters, vol. 96, no. 18, Article ID 181602, 2006.

[42] S. Ryu and T. Takayanagi, "Aspects of holographic entanglement entropy," Journal of High Energy Physics, vol. 8, p. 45, 2006. 

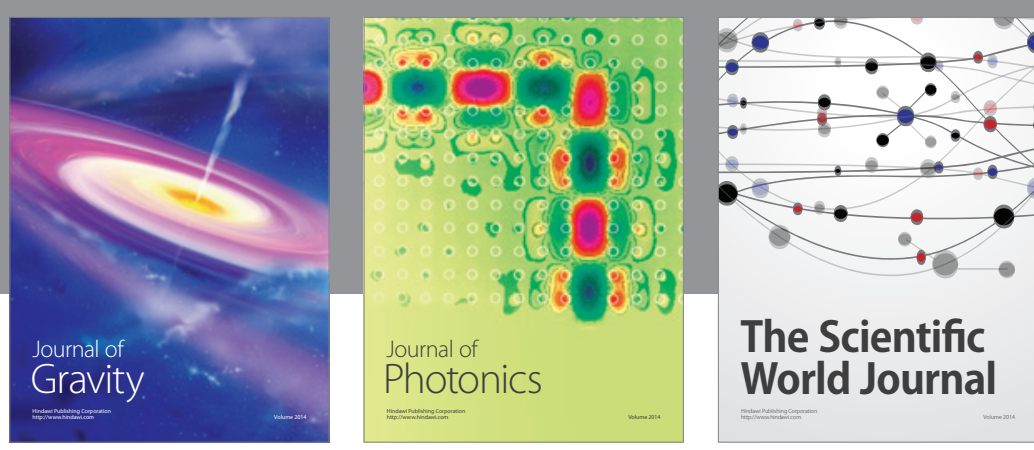

The Scientific World Journal
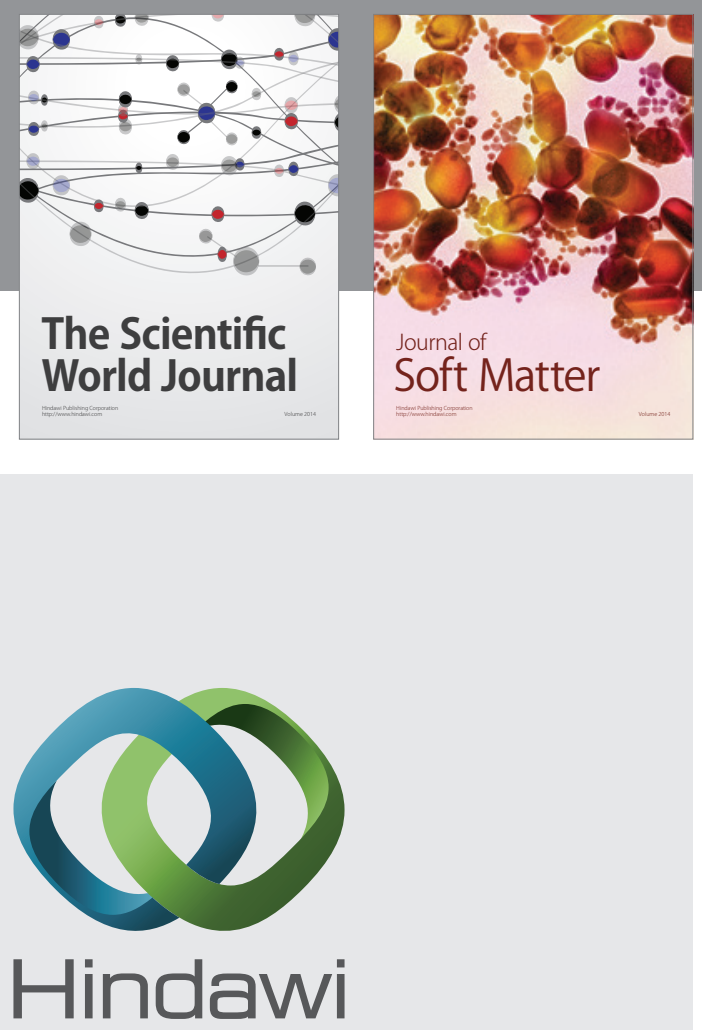

Submit your manuscripts at

http://www.hindawi.com

nternational Journal of

Statistical Mechanics
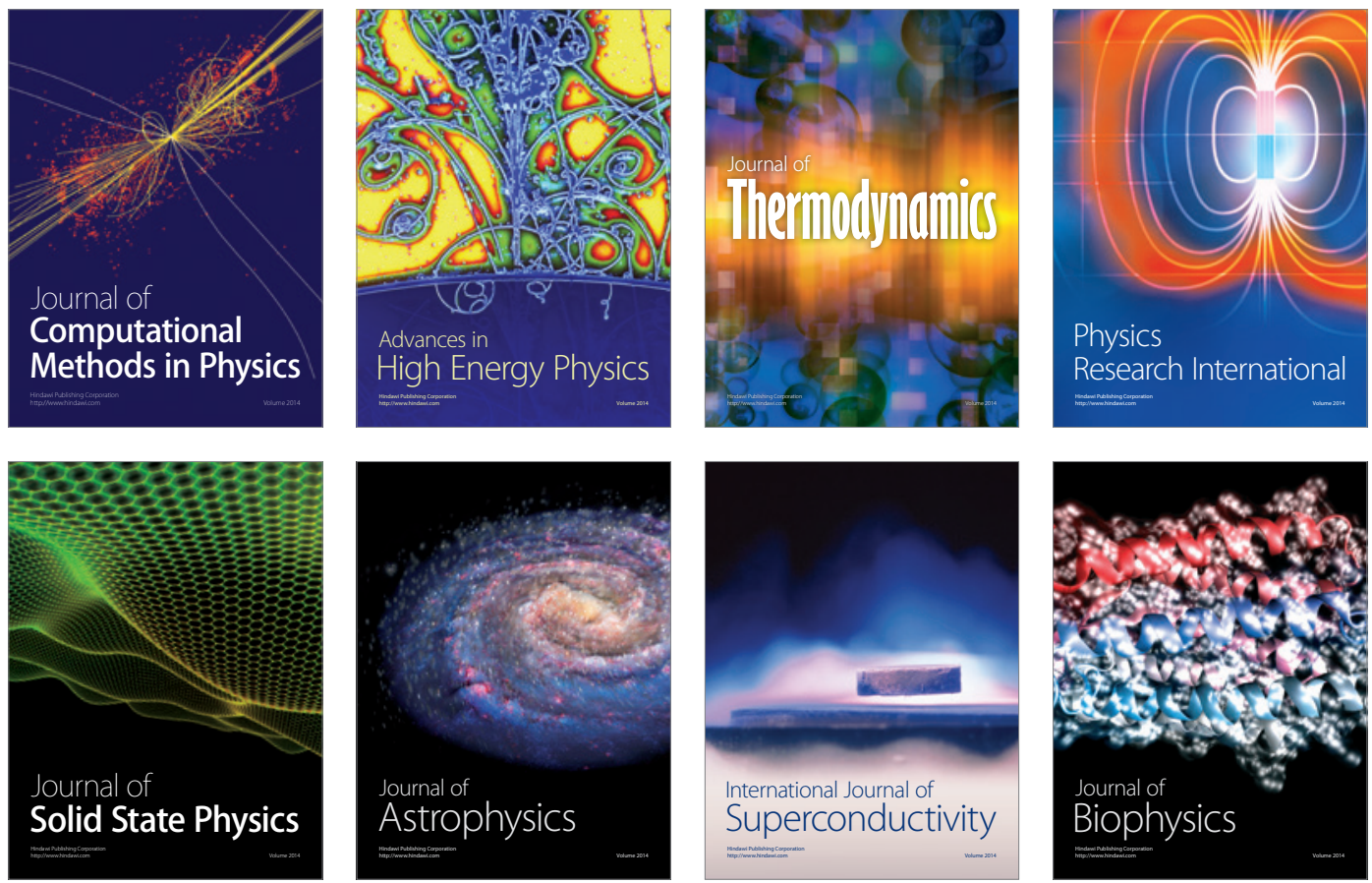
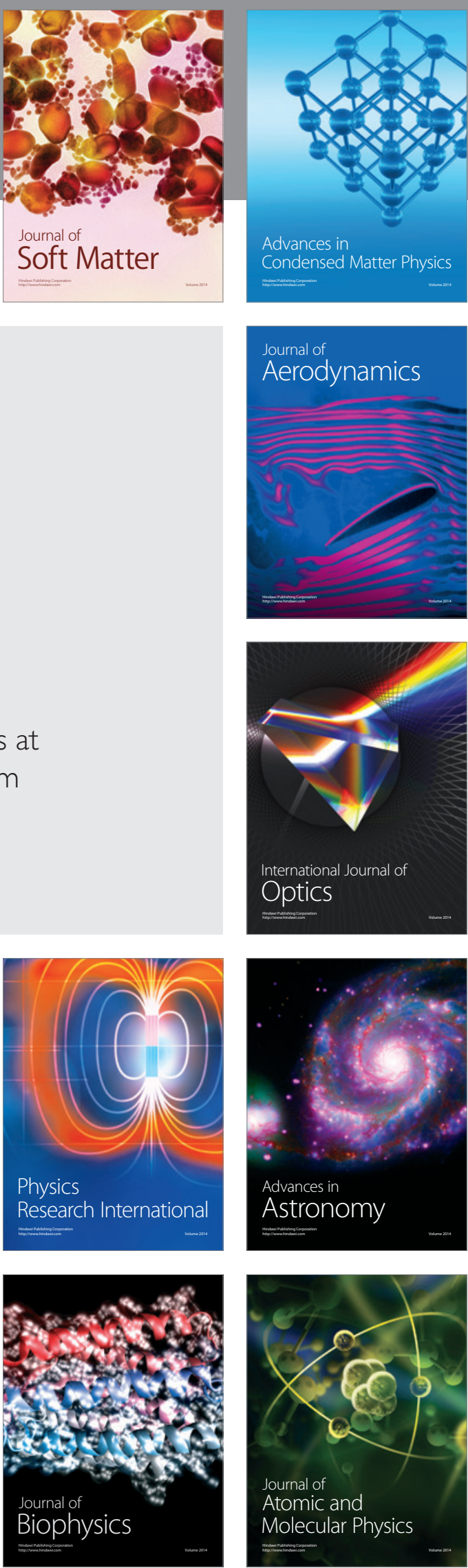